\title{
STRONG MAXIMUM PRINCIPLES FOR PARABOLIC NONLINEAR PROBLEMS WITH NONLOCAL INEQUALITIES TOGETHER WITH INTEGRALS
}

\author{
Ludwik Byszewski \\ Department of Applied Mathematics \\ Florida Institute of Technology \\ 150 West University Boulevard \\ Melbourne, Florida 32901-6988 \\ U.S.A.
}

\begin{abstract}
In [4] and [5], the author studied strong maximum principles for nonlinear parabolic problems with initial and nonlocal inequalities, respectively. Our purpose here is to extend results in [4] and [5] to strong maximum principles for nonlinear parabolic problems with nonlocal inequalities together with integrals. The results obtained in this paper can be applied in the theories of diffusion and heat conduction, since considered here integrals in nonlocal inequalities can be interpreted as mean amounts of the diffused substance or mean temperatures of the investigated medium.
\end{abstract}

Key words: Strong maximum principle, parabolic nonlinear systems, functional-differential inequalities, nonlocal inequalities.

AMS(MOS) Subject Classification Codes: 35B50, 35K55, 35R45, 35K99.

\section{INTRODUCTION}

In this paper we give a theorem on strong maximum principles for problems with a diagonal system of nonlinear parabolic functional-differential inequalities and with nonlocal inequalities together with integrals. The diagonal system of the inequalities considered here is of the following form:

$$
u_{t}^{i}(t, x) \leq f^{i}\left(t, x, u(t, x), u_{x}^{i}(t, x), u_{x x}^{i}(t, x), u\right) \quad(i=1, \ldots, m),
$$

where $(t, x) \in \mathrm{D} \subset\left(t_{0}, t_{0}+T\right] \times R^{n}$ and $\mathrm{D}$ is one of six relatively arbitrary sets more general than the cylindrical domain $\left(t_{0}, t_{0}+T\right] \times \mathrm{D}_{0} \subset R^{n+1}$. The symbol $u$ denotes the mapping

$$
u: \widetilde{\mathrm{D}} \ni(t, x) \rightarrow u(t, x)=\left(u^{1}(t, x), \ldots, u^{m}(t, x)\right) \in R^{m},
$$

where $\widetilde{\mathrm{D}}$ is an arbitrary set contained in $\left(-\infty, t_{0}+T\right] \times R^{n}$ such that $\overline{\mathrm{D}} \subset \widetilde{\mathrm{D}}$. The right-hand sides $f^{i}(i=1, \ldots, m)$ of system (1.1) are functionals of $u ; u_{x}^{i}(t, x)=\operatorname{grad}_{x} u^{i}(t, x)$ $(i=1, \ldots, m)$ and $u_{x x}^{i}(t, x)(i=1, \ldots, m)$ denote the matrices of second order derivatives with respect to $x$ of $u^{i}(t, x)(i=1, \ldots, m)$.

The nonlocal inequalities together with integrals, considered here, are of the form: 


$$
\begin{aligned}
& {\left[w^{j}\left(t_{0}, x\right)-K^{j}\right]+\sum_{i \in \mathrm{I}_{*}} h_{i}(x)\left[\frac{1}{\frac{1}{T_{2 \mathrm{i}}-T_{2 i-1}}} \quad \int_{T_{2 i-1} u^{j}(\tau, x) d \tau-K^{j}}^{T_{2 i}}\right] \leq 0} \\
& \text { for } x \in S_{t_{0}}(j=1, \ldots, m) \text {, }
\end{aligned}
$$

where $K^{j}(j=1, \ldots, m)$ are some constants, $I_{*}$ is a subset of a countable set $I$ of natural indices, $t_{0}<T_{2 i-1}<T_{2 i} \leq t_{0}+T(i \in I), h_{i}: S_{t_{0}} \rightarrow(-\infty, 0]\left(i \in \mathrm{I}_{*}\right)$ are some functions and

$$
S_{t_{0}}:=\operatorname{int}\left\{x \in R^{n}:\left(t_{0}, x\right) \in \overline{\mathrm{D}}\right\} .
$$

The results obtained in this paper are a continuation and direct generalizations of those given by the author [5] and [4]. Moreover, some results obtained here are direct generalizations of results given by Chabrowski [7]. Finally, some results obtained in the paper are indirect generalizations of those given by Chabrowski [6], Walter [15] and [16], Besala [2], Szarski [14], and Redheffer and Walter [13]. The method of the proof of the main theorem in this paper is similar to the method used in [5], and for ease in comparison of these methods we use in this article similar notation as in [5]. If the nonlocal inequalities considered here are initial inequalities, then the results obtained in this paper are reduced to those from [4] and are based on the publication of the author [3].

Parabolic problems with nonlocal conditions together with integrals were also investigated by Day [8], Friedman [9], Nakhusheva [12] and Kawohl [11]. However, considered in publications [8], [9], [12] and [11] both the nonlocal conditions and the integrals in those conditions are different from the nonlocal conditions (1.2) and the integrals in these conditions, respectively.

\section{PRELIMINARIES}

The notation and definitions given in this section are valid throughout this paper. We use the following notation: $R=(-\infty, \infty), R_{-}=(-\infty, 0], N=\{1,2, \ldots\}, x=\left(x_{1}, \ldots, x_{n}\right)$ $(n \in N)$.

For any vectors $z=\left(z^{1}, \ldots, z^{m}\right) \in R^{m}, \tilde{z}=\left(\tilde{z}^{1}, \ldots, \tilde{z}^{m}\right) \in R^{m}$ we write

$$
z \leq \tilde{z} \quad \text { if } \quad z^{i} \leq \tilde{z}^{i} \quad(i=1, \ldots, m) .
$$

Let $t_{0}$ be a real finite number and let $0<T<\infty$. A set $\mathrm{D} \subset\left\{(t, x): t>t_{0}, x \in R^{n}\right\}$ (bounded or unbounded) is called a set of type (P) if:

1. The projection of the interior of $\mathrm{D}$ on the $t$-axis is the interval $\left(t_{0}, t_{0}+T\right)$.

2. For every $(\tilde{t}, \tilde{x}) \in \mathrm{D}$ there is a positive $r$ such that

$$
\left\{(t, x):(t-\tilde{t})^{2}+\sum_{i=1}^{n}\left(x_{i}-\tilde{x}_{i}\right)^{2}<r, t<\tilde{t}\right\} \subset \mathrm{D}
$$


For any $t \in\left[t_{0}, t_{0}+T\right]$ we define the following sets:

and

$$
S_{t}=\left\{\begin{array}{l}
\text { int }\left\{x \in R^{n}:\left(t_{0}, x\right) \in \overline{\mathrm{D}}\right\} \text { for } t=t_{0}, \\
\left\{x \in R^{n}:(t, x) \in \mathrm{D}\right\} \text { for } t \neq t_{0}
\end{array}\right.
$$

$$
\delta_{t}=\left\{\begin{array}{l}
\operatorname{int}\left[\overline{\mathrm{D}} \cap\left(\left\{t_{0}\right\} \times R^{n}\right)\right] \text { for } t=t_{0}, \\
\mathrm{D} \cap\left(\{t\} \times R^{n}\right) \text { for } t \neq t_{0} .
\end{array}\right.
$$

It is easy to see, by condition 2 of the definition of a set of type (P), that $S_{t}$ and $\delta_{t}$ are open sets in $R^{n}$ and $R^{n+1}$, respectively.

Let $\tilde{\mathrm{D}}$ be a set contained in $\left(-\infty, t_{0}+T\right] \times R^{n}$ such that $\overline{\mathrm{D}} \subset \overline{\mathrm{D}}$. We introduce the following sets:

$$
\partial_{p} \mathrm{D}:=\widetilde{\mathrm{D} V} \quad \text { and } \quad \Gamma:=\partial_{p} \mathrm{D} \backslash \delta_{t_{0}} .
$$

For an arbitrary fixed point $(\tilde{t}, \tilde{x}) \in \mathrm{D}$ we denote by $S^{-}(\tilde{t}, \tilde{x})$ the set of points $(t, x) \in \mathrm{D}$ that can be joined with $(\tilde{t}, \tilde{x})$ by a polygonal line contained in $\mathrm{D}$ along which the $t$-coordinate is weakly increasing from $(t, x)$ to $(\tilde{t}, \tilde{x})$.

By $Z_{m}(\widetilde{D})$ we denote the space of continuous in $\overline{\mathrm{D}}$ mappings

$$
w: \tilde{\mathrm{D}} \ni(t, x) \rightarrow w(t, x)=\left(w^{1}(t, x), \ldots, w^{m}(t, x)\right) \in R^{m} .
$$

In the set of mappings bounded from above in $\widetilde{\mathrm{D}}$ and belonging to $Z_{m}(\widetilde{D})$ we define the functional

$$
[w]_{t}=\max _{i=1 \ldots m} \sup \left\{0, w^{i}(\tilde{t}, x):(\tilde{t}, x) \in \tilde{D}, \tilde{t} \leq t\right\}, \text { where } t \leq t_{0}+T .
$$

By $X$ we denote a fixed subset (not necessarily a linear subspace) of $Z_{m}(\widetilde{D})$ and by $M_{n \times n}(R)$ we denote the space of real square symmetric matrices $r=\left[r_{j k}\right]_{n \times n}$.

A mapping $u \in X$ is called regular in D if $u_{t}^{i}, u_{x}^{i}=\operatorname{grad}_{x} u^{i}, u_{x x}^{i}=\left[u_{x_{j} x_{k}}^{i}\right]_{n \times n}(i=1, \ldots, m)$ are continuous in D.

Let the mappings

$f: \mathrm{D} \times R^{m} \times R^{n} \times M_{n \times n}(R) \times Z_{m}(\widetilde{\mathrm{D}}) \ni(t, x, z, q, r, w) \rightarrow f^{i}(t, x, z, q, r, w) \in R(i=1, \ldots, m)$

be given and let the operators $P_{i}(i=1, \ldots, m)$ be defined by the formulae

$$
P_{i} u(t, x)=u_{t}^{i}(t, x)-f^{i}\left(t, x, u(t, x), u_{x}^{i}(t, x), u_{x x}^{i}(t, x), u\right), u \in X,(t, x) \in \mathrm{D}(i=1, \ldots, m) .
$$


A regular mapping $u$ in $\mathrm{D}$ is called solution of the system of the functional-differential inequalities

$$
P_{i} u(t, x) \leq 0, \quad(t, x) \in \mathrm{D} \quad(i=1, \ldots, m)
$$

in D if (2.1) is satisfied.

For any set $Z \subset \widetilde{D}$ and for a mapping $u \in X$ we use the symbol $\max _{(t, x) \in Z} u(t, x)$ in the sense:

$$
\left(\max _{(t, x) \in Z} u^{1}(t, x), \ldots, \max _{(t, x) \in Z} u^{m}(t, x)\right) .
$$

Let us define the following set:

$$
\mathcal{S}=\bigcup_{i \in \mathrm{I}}\left(\delta_{T_{2 i-1}} \cup \delta_{T_{2 i}}\right)
$$

where $I$ is a countable set of all such mutually different natural numbers that:

(i) $t_{0}<T_{2 i-1}<T_{2 i} \leq t_{0}+T$ for $i \in \mathrm{I}$ and $T_{2 i-1} \neq T_{2 j-1}, T_{2 i} \neq T_{2 j}$ for $i, j \in I, i \neq j$,

(ii) $T_{0}:=\inf \left\{T_{2 i-1}: i \in I\right\}>t_{0}$ if card $\mathrm{I}=\aleph_{0}$,

(iii) $S_{t} \supset S_{t_{0}}$ for every $t \in \underset{i \in I}{\cup}\left[T_{2 i-1}, T_{2 i}\right]$,

(iv) $\quad S_{t} \supset S_{\mathrm{t}_{0}}$ for every $t \in\left[T_{0}, t_{0}+T\right]$ if card $\mathrm{I}=\aleph_{0}$.

An unbounded set $\mathrm{D}$ of type $(\mathrm{P})$ is called a set of type $\left(\mathrm{P}_{\mathrm{S} \Gamma}\right)$ (see Fig.1) if:

(a) $\mathcal{S} \neq \varnothing$,

(b) $\quad \Gamma \cap \bar{\delta}_{t_{0}} \neq \varnothing$

Let $\mathcal{S}_{*}$ denote a non-empty subset of $\mathcal{S}$. We define the following set:

$$
\mathrm{I}_{*}=\left\{i \in I:\left(\delta_{T_{2 i-1}} \cup \delta_{T_{2 i}}\right) \subset \mathcal{S}_{*}\right\} .
$$

A bounded set $\mathrm{D}$ of type (P) satisfying condition (a) of the definition of a set of type $\left(\mathrm{P}_{\mathrm{S} \Gamma}\right)$ is called a set of type $\left(\mathrm{P}_{\mathrm{SB}}\right)$. 
${ }^{t} \Lambda$

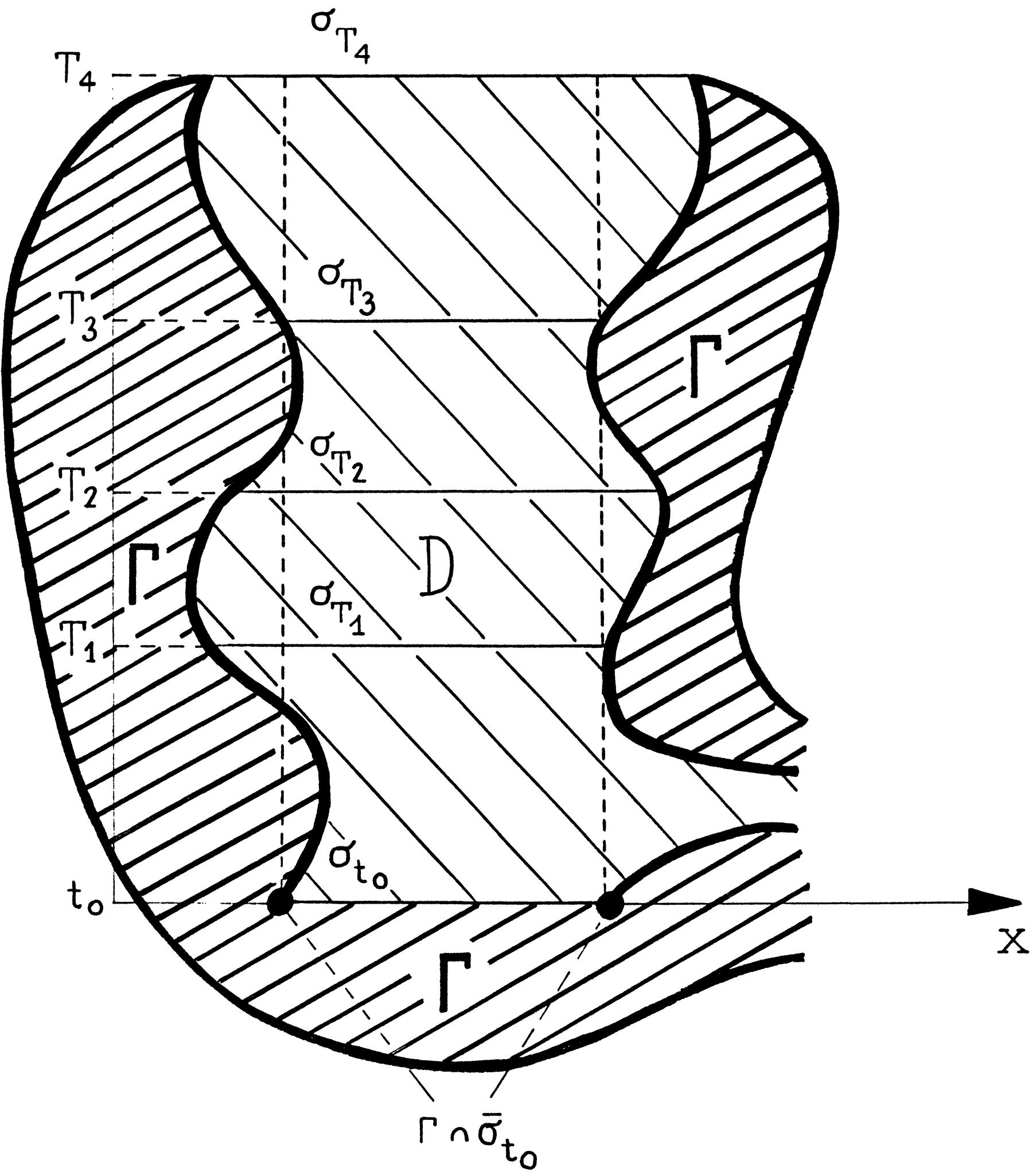

Figure 1. The set $\mathrm{D}$ of type $\left(\mathrm{P}_{\mathrm{S} \Gamma}\right)$ if $\mathrm{D}=($ int $\mathrm{D}) \cup \delta_{t_{0}+T}, I=\{1,2,3,4\}$ and $t_{0}<T_{1}<T_{2}<T_{3}<T_{4}=t_{0}+T$ 
It is easy to see that if $\mathrm{D}$ is a set of type $\left(\mathrm{P}_{\mathrm{SB}}\right)$, then $\mathrm{D}$ satisfies condition $(\mathrm{b})$ of the definition of a set of type $\left(\mathrm{P}_{\mathrm{S} \Gamma}\right)$. Moreover, it is obvious that if $\mathrm{D}_{0}$ is a bounded subset [ $\mathrm{D}_{0}$ is an unbounded essential subset] of $R^{n}$, then $\mathrm{D}=\left(t_{0}, t_{0}+T\right] \times \mathrm{D}_{0}$ is a set of type $\left(\mathrm{P}_{\mathrm{SB}}\right)\left[\left(\mathrm{P}_{\mathrm{Sr}}\right)\right.$, respectively].

\section{STRONG MAXIMUM PRINCIPLES WITH NONLOCAL INEQUALITIES TOGETHER WITH INTEGRALS IN SETS OF TYPES $\left(\mathrm{P}_{\mathrm{S} \Gamma}\right)$ AND $\left(\mathrm{P}_{\mathrm{SB}}\right)$.}

Our main result is the following theorem on strong maximum principles with nonlocal inequalities together with integrals in sets of types $\left(\mathrm{P}_{\mathrm{S} \Gamma}\right)$ and $\left(\mathrm{P}_{\mathrm{SB}}\right)$ :

Theorem 3.1: Assume that:

(1) $\mathrm{D}$ is a set of type $\left(\mathrm{P}_{\mathrm{S} \Gamma}\right)$ or $\left(\mathrm{P}_{\mathrm{SB}}\right)$.

(2) The mappings $f^{i}(i=1, \ldots, m)$ are weakly increasing with respect to $z^{1}, \ldots, z^{i-1}, z^{i+1}, \ldots, z^{m}(i=1, \ldots, m)$. Moreover, there is a positive constant $L$ such that the inequalities

$$
\begin{gathered}
f^{i}(t, x, z, q, r, w)-f^{i}(t, x, \tilde{z}, \tilde{q}, \tilde{r}, \tilde{w}) \\
\leq L\left(\max _{k=1 \ldots m}\left|z^{k}-\tilde{z}^{k}\right|+\left.|x| \sum_{j=1}^{n}\left|q^{j}-\tilde{q}^{j \mid}+\right| x\right|^{2} \sum_{j ; k=1}^{n}\left|r_{j k}-\tilde{r}_{j k}\right|+[w-\tilde{w}]_{t}\right)
\end{gathered}
$$

are satisfied for all $(t, x) \in \mathrm{D}, z, \tilde{z} \in R^{m}, q, \tilde{q} \in R^{n}, r, \tilde{r} \in M_{n \times n}(R), w, \tilde{w} \in X$, $\sup _{(t, x) \in \overline{\mathrm{D}}}[w(t, x)-\tilde{w}(t, x)]<\infty(i=1, \ldots, m)$.

(3) The mapping $u$ belongs to $X$ and the maximum of $u$ on $\Gamma$ is attained. Moreover,

$$
K=\left(K^{1}, \ldots, K^{m}\right):=\max _{(t, x) \in \Gamma} u(t, x)
$$

and $K \in X$.

(4) The inequalities

$$
\left[u^{j}\left(t_{0}, x\right)-K^{j}\right]+\sum_{i \in I_{*}} h_{i}(x)\left[\frac{1}{T_{2 i}-T_{2 i-1}} \int_{T_{2 i-1}}^{T_{2 i}} u^{j}(\tau, x) d \tau-K^{j}\right] \leq 0 \text { for } x \in S_{t_{0}}(j=1, \ldots, m)
$$

are satisfied, where $h_{i}: S_{t_{0}} \rightarrow R_{-}\left(i \in \mathrm{I}_{*}\right)$ are given functions such that $-1 \leq \sum_{i \in \mathrm{I}_{*}} h_{\mathrm{i}}(x) \leq 0$ for $x \in S_{t_{0}}$ and, additionally, if card $\mathrm{I}_{*}=\aleph_{0}$, then the series $\sum_{i \in \mathrm{I}_{*}} \frac{h_{i}(x)}{T_{2 i}-T_{2 i-1}} \int_{2 i-1}^{T_{2 i}} u(\tau, x) d \tau$ $(j=1, \ldots, m)$ are convergent for $x \in S_{t_{0}}$.

(5) The maximum of $u$ in $\widetilde{\mathrm{D}}$ is attained. Moreover, 


$$
M=\left(M^{1}, \ldots, M^{m}\right):=\max _{(t, x) \in \mathrm{D}} u(t, x)
$$

and $M \in X$.

(6) The inequalities

are satisfied.

$$
f^{i}(t, x, M, 0,0, M) \leq 0 \quad \text { for } \quad(t, x) \in \mathrm{D} \quad(i=1, \ldots, m)
$$

(7) The mapping $u$ is a solution of system (2.1) in D.

(8) The mappings $f^{i}(i=1, \ldots, m)$ are parabolic with respect to $u$ in $\mathrm{D}$ and uniformly parabolic with respect to $M$ in any compact subset of $\mathrm{D}$ (cf. [3] or [4]).

Then

$$
\max _{(t, x) \in \tilde{D}} u(t, x)=\max _{(t, x) \in \Gamma} u(t, x) .
$$

Moreover, if there is a point $(\tilde{t}, \tilde{x}) \in \mathrm{D}$ such that $u(\tilde{t}, \tilde{x})=\max _{(t, x) \in \mathrm{D}} u(t, x)$, then

$$
u(t, x)=\max _{(t, x) \in \Gamma^{u}} u(t, x) \text { for }(t, x) \in S^{-}(\tilde{t}, \tilde{x}) .
$$

Proof: We shall prove Theorem 3.1 for a set of type $\left(\mathrm{P}_{\mathrm{S} \Gamma}\right)$ only since the proof of this theorem for a set of type $\left(\mathrm{P}_{\mathrm{SB}}\right)$ is analogous.

Since each set of type $\left(\mathrm{P}_{\mathrm{S} \Gamma}\right)$ is a set of type $\left(\mathrm{P}_{\mathrm{Z \Gamma}}\right)$ from [5] then, in the case if $\sum_{i \in I_{*}} h_{i}(x)=0$ for $x \in S_{t_{0}}$, Theorem 3.1 from this paper is a consequence of Theorem 3.1 of [5]. Therefore, we shall prove Theorem 3.1 only in the case if the following condition holds:

$$
-1 \leq \sum_{i \in \mathrm{I}_{*}} h_{i}(x)<0 \text { for } x \in S_{t_{0}}
$$

Assume, so, (3.5) holds and, since we shall argue by contradiction, suppose

$$
M \neq K \text {. }
$$

But, from (3.1) and (3.3), we have

$$
K \leq M \text {. }
$$

Consequently, by (3.6) and (3.7), we obtain

$$
K<M \text {. }
$$

Observe, from assumption (5), that the following condition holds:

$$
\text { There is }\left(t^{*}, x^{*}\right) \in \widetilde{D} \text { such that } u\left(t^{*}, x^{*}\right)=M:=\max _{(t, x) \in \mathrm{D}} u(t, x) .
$$


By (3.9), by assumption (3) and by (3.8), we have

$$
\left(t^{*}, x^{*}\right) \in \widetilde{\mathrm{D}} \backslash \Gamma=\mathrm{D} \cup \delta_{t_{0}} .
$$

An analogous argument as in the proof of Theorem 4.1 from [4] yields

$$
\left(t^{*}, x^{*}\right) \notin \mathrm{D}
$$

Conditions (3.10) and (3.11) give

$$
\left(t^{*}, x^{*}\right) \in \delta_{t_{0}} .
$$

Simultaneously, by the definitions of sets $\mathrm{I}$ and $\mathrm{I}_{*}$, we must consider the following cases:

(A) $\quad \mathrm{I}_{*}$ is a finite set, i.e., without loss of generality there is a number $p \in N$ such that $\mathrm{I}_{*}=\{1, \ldots, p\}$.

(B) $\quad \operatorname{card} \mathrm{I}_{*}=\kappa_{0}$.

First we shall consider case (A). And so, by (3.2) and by the inequality

$$
u\left(t, x^{*}\right)<u\left(t_{0}, x^{*}\right) \quad \text { for } t \in \bigcup_{i=1}^{p}\left[T_{2 i-1}, T_{2 i}\right],
$$

being a consequence of (3.9), (3.12), and of (a)(i), (a)(iii) of the definition of a set of type $\left(\mathrm{P}_{\mathrm{S} \Gamma}\right)$, we have

$$
\begin{aligned}
& 0 \geq\left[w^{j}\left(t_{0}, x^{*}\right)-K^{j}\right]+\sum_{i=1}^{p} h_{i}\left(x^{*}\right)\left[\frac{1}{T_{2 \mathrm{i}}-T_{2 i-1}} \int_{2 i-1}^{T_{2 i}} w^{j}\left(\tau, x^{*}\right) d \tau-K^{j}\right] \\
& \geq\left[w^{j}\left(t_{0}, x^{*}\right)-K^{j}\right]+\sum_{i=1}^{p} h_{i}\left(x^{*}\right)\left[\frac{1}{T_{2 i}-T_{2 i-1}} \int_{2 \mathrm{i}-1}^{T_{2 i}} w^{j}\left(t_{0}, x^{*}\right) d \tau-K^{j}\right] \\
& =\left[w^{j}\left(t_{0}, x^{*}\right)-K^{j}\right] \cdot\left[1+\sum_{i=1}^{p} h_{i}\left(x^{*}\right)\right] \quad(j=1, \ldots, m) .
\end{aligned}
$$

Hence

$$
u\left(t_{0}, x^{*}\right) \leq K \quad \text { if } \quad 1+\sum_{i=1}^{p} h_{i}\left(x^{*}\right)>0 .
$$

Then, from (3.8) and (3.12), we obtain a contradiction of (3.13) with (3.9). Assume now 


$$
\sum_{i=1}^{p} h_{i}\left(x^{*}\right)=-1
$$

By the mean-value integral theorem we have that for every $j \in\{1, \ldots, m\}$ and $i \in\{1, \ldots, p\}$ there is $\widetilde{T}_{i}^{j} \in\left[T_{2 i-1}, T_{2 i}\right]$ such that

$$
u^{j}\left(\widetilde{T}_{i}^{j}, x^{*}\right)=\frac{1}{T_{2 i}-T_{2 i-1}} \int_{T_{2 i-1}}^{T_{2 i}} u^{j}\left(\tau, x^{*}\right) d \tau .
$$

Simultaneously, for every $j \in\{1, \ldots, m\}$ there is a number $l_{j} \in\{1, \ldots, p\}$ such that

$$
w^{j}\left(\tilde{T}_{l_{j}}^{j}, x^{*}\right)=\max _{i=1 \ldots p} u^{j}\left(\tilde{T}_{i}^{j}, x^{*}\right) .
$$

Consequently, by (3.14), (3.16), (3.15) and (3.2), we obtain

$$
\begin{aligned}
w^{j}\left(t_{0}, x^{*}\right)-w^{j}\left(\widetilde{T}_{l_{j}}^{j} x^{*}\right)= & {\left[w\left(t_{0}, x^{*}\right)-K^{j}\right]-\left[w^{j}\left(\widetilde{T}_{l_{j}}^{j} x^{*}\right)-K^{j}\right] } \\
& =\left[w^{j}\left(t_{0}, x^{*}\right)-K^{j}\right]+\sum_{i=1}^{p} h_{i}\left(x^{*}\right)\left[u^{j}\left(\widetilde{T}_{l_{j}}^{j}, x^{*}\right)-K^{j}\right] \\
& \leq\left[w^{j}\left(t_{0}, x^{*}\right)-K^{j}\right]+\sum_{i=1}^{p} h_{i}\left(x^{*}\right)\left[w^{j}\left(\widetilde{T}_{i}^{j}, x^{*}\right)-K^{j}\right] \\
& =\left[w^{j}\left(t_{0}, x^{*}\right)-K^{j}\right]+\sum_{i=1}^{p} h_{i}\left(x^{*}\right)\left[\frac{1}{T_{2 i}-T_{2 i-1}} \int_{T_{2 i-1}}^{T_{2 i}} u^{j}\left(\tau, x^{*}\right) d \tau-K^{j}\right] \\
& \leq 0 \quad(j=1, \ldots, m) .
\end{aligned}
$$

Hence

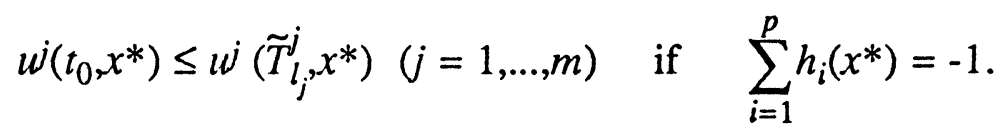

Since, by $(\mathrm{a})(i)$ of the definition of a set of type $\left(\mathrm{P}_{\mathrm{S} \Gamma}\right), \tilde{T}_{l_{j}}^{j}>t_{0}(j=1, \ldots, m)$, we get from (3.12) that condition (3.17) is at a contradiction with condition (3.9). This completes the proof of (3.4) if $I_{*}$ is a finite set. 
It remains to investigate case (B). Analogously as in the proof of (3.4) in case (A), by assumption (4) and by the inequality

$$
u\left(t, x^{*}\right)<u\left(t_{0}, x^{*}\right) \quad \text { for } \quad t \in \bigcup_{i \in I_{*}}\left[T_{2 i-1}, T_{2 i}\right]
$$

being a consequence of (3.9), (3.12), and of (a)(i), (a)(iii) of the definition of a set type $\left(\mathrm{P}_{\mathrm{S} \Gamma}\right)$, we have

$$
\begin{aligned}
& 0 \geq\left[w^{j}\left(t_{0}, x^{*}\right)-K^{j}\right]+\sum_{i \in \mathrm{I}_{*}} h_{i}\left(x^{*}\right)\left[\frac{1}{\frac{1}{T_{2 i}-T_{2 i-1}}} \quad \int_{T_{2 i-1}}^{T_{2 i}} u\left(\tau, x^{*}\right) d \tau-K^{j}\right] \\
& \geq\left[w^{j}\left(t_{0}, x^{*}\right)-K^{j}\right]+\sum_{i \in \mathrm{I}_{*}} h_{i}\left(x^{*}\right)\left[\frac{1}{\frac{1}{T_{2 i}-T_{2 i-1}}} \quad \int_{T_{2 i-1}}^{T_{2 i}} u^{j}\left(t_{0}, x^{*}\right) d \tau-K^{j}\right] \\
& =\left[w^{j}\left(t_{0}, x^{*}\right)-K^{j}\right] \cdot\left[1+\sum_{i \in \mathrm{I}_{*}} h_{i}\left(x^{*}\right)\right] \quad(j=1, \ldots, m) .
\end{aligned}
$$

Hence

$$
u\left(t_{0}, x^{*}\right) \leq K \quad \text { if } \quad 1+\sum_{i \in I_{*}} h_{i}\left(x^{*}\right)>0
$$

Then, from (3.8) and (3.12), we obtain a contradiction of (3.18) with (3.9). Assume now

$$
\sum_{i \in I_{*}} h_{i}\left(x^{*}\right)=-1
$$

By the mean-value integral theorem we have that for every $j \in\{1, \ldots, m\}$ and $i \in \mathrm{I}_{*}$ there is $\tilde{T}_{i}^{j} \in\left[T_{2 i-1}, T_{2 i}\right]$ such that

$$
w^{j}\left(\tilde{T}_{l_{j}}^{j} x^{*}\right)=\frac{1}{T_{2 i}-T_{2 i-1}} \int_{T_{2 i-1}}^{T_{2 i}} w^{j}\left(\tau, x^{*}\right) d \tau .
$$

Let

$$
\widetilde{T}_{*}^{j}=\inf _{i \in \mathrm{I}_{*}} \widetilde{T}_{i}^{j}(j=1, \ldots, m)
$$

Since $u \in C(\overline{\mathrm{D}})$ and since, by (3.12) and by (a)(iv), (a)(ii) of the definition of a set type $\left(\mathrm{P}_{\mathrm{S} \Gamma}\right), x^{*} \in S_{t}$ for every $t \in\left[T_{0}, t_{0}+T\right]$ if card $\mathrm{I}=\kappa_{\mathrm{O}}$, it follows from (3.21) that for every $j \in\{1, \ldots, m\}$ there is a number $\hat{t}_{j} \in\left[\tilde{T}_{*}^{j} t_{0}+T\right]$ such that 


$$
u^{j}\left(\hat{t}_{j}, x^{*}\right)=\max _{\left[\tilde{T}_{*}^{j}, t_{0}+T\right]} u^{j}\left(t, x^{*}\right) .
$$

Consequently, by (3.19), (3.22), (3.20) and by assumption (4), we obtain

$$
\begin{aligned}
& w^{j}\left(t_{0}, x^{*}\right)-w^{j}\left(\hat{t}_{j}, x^{*}\right)=\left[w^{j}\left(t_{0}, x^{*}\right)-K^{j}\right]-\left[w^{j}\left(\hat{t}_{j}, x^{*}\right)-K^{j}\right] \\
& =\left[w^{j}\left(t_{0}, x^{*}\right)-K^{j}\right]+\sum_{i \in \mathrm{I}_{*}} h_{i}\left(x^{*}\right)\left[w^{j}\left(\hat{t}_{j}, x^{*}\right)-K^{j}\right] \\
& \leq\left[u^{j}\left(t_{0}, x^{*}\right)-K^{j}\right]+\sum_{i \in \mathrm{I}_{*}} h_{i}\left(x^{*}\right)\left[u^{\left.j\left(\tilde{T}_{i}^{j}, x^{*}\right)-K^{j}\right]}\right. \\
& =\left[w^{j}\left(t_{0}, x^{*}\right)-K^{j}\right]+\sum_{i \in \mathrm{I}_{*}} h_{i}\left(x^{*}\right)\left[\frac{1}{\frac{1}{T_{2 i}-T_{2 i-1}}} \quad \int_{T_{2 i-1}}^{T_{2 i}}\left(\tau, x^{*}\right) d \tau-K^{j}\right] \\
& \leq 0 \quad(j=1, \ldots, m) .
\end{aligned}
$$

Hence

$$
w^{i}\left(t_{0}, x^{*}\right) \leq w^{j}\left(\hat{t}_{\mathrm{j}}, x^{*}\right) \quad(j=1, \ldots, m) \quad \text { if } \sum_{i \in \mathrm{I}_{*}} h_{i}\left(x^{*}\right)=-1
$$

Since, by (a) (ii) of the definition of a set of type $\left(\mathrm{P}_{\mathrm{S} \Gamma}\right), \hat{t}_{j}>t_{0}(j=1, \ldots, m)$, we get from (3.12) that condition (3.23) is at a contradiction with condition (3.9). This completes the proof of equality (3.4).

The second part of Theorem 3.1 is a consequence of equality (3.4) and of Lemma 3.1 from [4]. Therefore, the proof of Theorem 3.1 is complete.

\section{REMARKS}

Remark 4.1. It is easy to see, by the proof of Theorem 3.1 from this paper and by the proofs of Theorems 3.1 and 4.1 from papers [5] and [4], respectively, that if the functions $h_{i}$ $\left(i \in \mathrm{I}_{*}\right.$ ) from assumption (4) of Theorem 3.1 satisfy the condition

$$
\left[\sum_{i \in \mathrm{I}_{*}} h_{i}(x)=0\right]-1<\sum_{i \in \mathrm{I}_{*}} h_{i}(x) \leq 0 \quad \text { for } \quad x \in S_{t_{0}}
$$

then it is sufficient to assume in this theorem that [D is only an unbounded set of type (P) satisfying condition (b) of the definition of a set of type $\left(\mathrm{P}_{S \Gamma}\right)$ or $\mathrm{D}$ is only a bounded set of type $(\mathrm{P})$, i.e., according to the terminology introduced in [4], $\mathrm{D}$ is a set of type $\left(\mathrm{P}_{\Gamma}\right)$ or $\left(\mathrm{P}_{\mathrm{B}}\right)$, respectively] $\mathrm{D}$ is only an unbounded set of type $(\mathrm{P})$ satisfying conditions (a)(i), (a)(iii) and $(b)$ of the definition of a set of type $\left(\mathrm{P}_{S \Gamma}\right)$ or $\mathrm{D}$ is only a bounded set of type $(\mathrm{P})$ 
satisfying conditions (a)(i) and (a)(iii) of the definition of a set of type $\left(\mathrm{P}_{\mathrm{S} \Gamma}\right)$. Moreover, if $I_{*}$ is a finite set and

$$
-1 \leq \sum_{i \in \mathrm{I}_{*}} h_{i}(x) \leq 0 \quad \text { for } \quad x \in S_{t_{0}},
$$

then it is sufficient to assume in Theorem 3.1 that $D$ is only an unbounded set of type (P) satisfying conditions (a)(i), (a)(iii) and (b) or D is only a bounded set of type (P) satisfying conditions (a) (i) and (a)(iii).

Remark 4.2. If $\mathrm{D}$ is a set of type $\left(\mathrm{P}_{\mathrm{SB}}\right)$ and if $\widetilde{\mathrm{D}}=\overline{\mathrm{D}}$, then the first part of assumption (3) of Theorem 3.1 relative to the maximum of $u$ and the first part of assumption (5) of this theorem are trivially satisfied since $u, v \in \mathrm{C}(\overline{\mathrm{D}})$ and $\Gamma$ is the bounded and closed set in this case.

Remark 4.3. Analogously as in [5] (cf. [5], Theorem 3.2) we can obtain a theorem on strong minimum principles with the following nonlocal inequalities together with integrals:

$$
\begin{gathered}
{\left[v^{j}\left(t_{0}, x\right)-k^{j}\right]+\sum_{i \in \mathrm{I}_{*}} h_{i}(x)\left[\frac{1}{\frac{1}{T_{2 i}-T_{2 i-1}}} \int_{T_{2 i-1}}^{T_{2 i}} v^{j}(\tau, x) d \tau-k^{j}\right] \geq 0} \\
\text { for } x \in S_{t_{0}}(j=1, \ldots, m)
\end{gathered}
$$

in sets of types $\left(\mathrm{P}_{\mathrm{S} \Gamma}\right)$ and $\left(\mathrm{P}_{\mathrm{SB}}\right)$.

\section{PHYSICAL INTERPRETATIONS OF PROBLEMS CONSIDERED.}

Theorem 3.1 can be applied to descriptions of physical phenomena in which we can measure sums of mean temperatures of substances or sums of mean amounts of substances according to the following formulae:

$$
w\left(t_{0}, x\right)+\sum_{i \in \mathrm{I}_{*}} \frac{h_{i}(x)}{T_{2 i}-T_{2 i-1}} \int_{T_{2 i-1}}^{T_{2 i}} w(\tau, x) d \tau \quad \text { for } x \in S_{t_{0}}(j=1, \ldots, m)
$$

( $h_{i}\left(i \in \mathrm{I}_{*}\right)$ are known functions). For example, Theorem 3.1 can be applied to the description of a diffusion phenomenon of a little amount of a gas in a transparent tube, under the assumption that the diffusion is observed by the surface of this tube. The measurement $u\left(t_{0}, x\right)(m=1)$ of small amount of the gas at the initial instant $t_{0}$ is usually less precise than the following measurement: 


$$
u\left(t_{0}, x\right)+\sum_{i \in I_{*}} \frac{h_{i}(x)}{T_{2 i}-T_{2 i-1}} \int_{T_{2 i-1}}^{T_{2 i}} u(\tau, x) d \tau \quad \text { for } \quad x \in S_{t_{\mathrm{o}}}(m=1)
$$

where

$$
\frac{1}{T_{2 i}-T_{2 i-1}} \int_{T_{2 i-1}}^{T_{2 i}} u(\tau, x) d \tau \quad \text { for } \quad x \in S_{t_{0}}\left(i \in \mathrm{I}_{*}, m=1\right)
$$

are the mean amounts of this gas on the intervals $\left[T_{2 i-1}, T_{2 i}\right]\left(i \in \mathrm{I}_{*}\right)$, respectively. Therefore, Theorem 3.1 seems to be more useful in some physical applications than Theorem 4.1 from [4] on strong maximum principles with initial inequalities of the form:

$$
u\left(t_{0}, x\right) \leq K \quad \text { for } \quad x \in S_{t_{0}}
$$

Let us observe that Theorem 3.1 from the paper is also more useful in some physical applications than Theorem 3.1 from [5], since considered here inequalities (3.2) are more sensitive to measurements than the following inequalities:

$$
\left[u^{j}\left(t_{0}, x^{*}\right)-K^{j}\right]+\sum_{i \in \mathrm{I}_{*}} h_{i}\left(x^{*}\right)\left[u^{j}\left(T_{i}, x\right)-K^{j}\right] \leq 0 \quad \text { for } \quad x \in S_{t_{0}} \quad(j=1, \ldots, m)
$$

given by the author in [5].

$$
\text { If } \mathrm{I}_{*}=\{1\}, T_{1}=t_{0}+T-\Delta t, 0<\Delta t<T, T_{2}=t_{0}+T,-1 \leq h_{i}(x)=-h(x) \leq 0 \text { for } x \in S_{t_{0}}
$$
and $m=1$, then the nonlocal conditions:

$$
u^{j}\left(t_{0}, x\right)+\sum_{i \in I_{*}} \frac{h_{i}(x)}{T_{2 i}-T_{2 i-1}} \int_{T_{2 i-1}}^{T_{2 i}} u^{j}(\tau, x) d \tau=0 \quad \text { for } \quad x \in S_{t_{0}} \quad(j=1, \ldots, m)
$$

are reduced to the following condition:

$$
u\left(t_{0}, x\right)=\frac{h(x)}{\Delta t} \int_{t_{0}+T-\Delta t}^{t_{0}+T} u(\tau, x) d \tau \quad \text { for } \quad x \in S_{t_{0}} \quad(m=1)
$$

and this condition can be used to the description of heat effects in atomic reactors. It is easy to see, by (5.1), that if $u\left(t_{0}, x\right)$ is interpreted as the given temperature in an atomic reactor at the initial instant $t_{0}$, then the atomic reaction is the safest for $1 \cong h(x) \leq 1$ and this reaction is the most dangerous for $0<h(x) \cong 0$. In the case if $h(x)=1$ for $x \in S_{t_{0}}$, formula (5.1) is reduced to the condition: 


$$
u\left(t_{0}, x\right)=\frac{1}{\Delta t} \int_{t_{0}+T-\Delta t}^{t_{0}+T} u(\tau, x) d \tau \quad \text { for } \quad x \in S_{t_{0}} \quad(m=1),
$$

which is the modification of the periodic condition:

$$
u\left(t_{0}, x\right)=u\left(t_{0}+T, x\right) \quad \text { for } \quad x \in S_{t_{0}} \quad(m=1),
$$

considered among other things by Beltramo and Hess [1] and Hess [10].

Remark 5.1. The considerations from Section 5 concerning Theorem 3.1 are also true for the strong minimum principles with nonlocal inequalities (4.1) (cf. Remark 4.3).

\section{REFERENCES}

[1] Beltramo, A., and P. Hess, On the principal eigenvalue of a periodic-parabolic operator, Comm. in Partial Differential Equations 9 (1984), 919-941.

[2] Besala, P., An extension of the strong maximum principle for parabolic equations, Bull. Acad. Polon. Sci., Sér. sci. math., astr. et phys. 19 (1971), 10031006.

[3] Byszewski, L., Strong maximum principle for implicit nonlinear parabolic functional-differential inequalities in arbitrary domains, Univ. Iagell. Acta Math. 24 (1984), 327-339.

[4] Strong maximum and minimum principles for parabolic functionaldifferential problems with initial inequalities $u\left(t_{0}, x\right) \leq(\geq) K$, Ann. Polon. Math. (to appear).

[5] Strong maximum and minimum principles for parabolic functionaldifferential problems with non-local inequalities $\left[w^{i}\left(t_{0}, x\right)-K^{j}\right]+$ $\sum_{i} h_{i}(x)\left[w^{i}\left(T_{i}, x\right)-K^{j}\right] \leq(\geq) 0$, Ann. Polon. Math. (to appear).

[6] Chabrowski, J., On non-local problems for parabolic equations, Nagoya Math. J. 93 (1984), 109-131.

[7] On the non-local problem with a functional for parabolic equation, Funkcialaj Ekvacioj 27 (1984), 101-123.

[8] Day, W.A., A decreasing property of solutions of parabolic equations with applications to thermoelasticity, Quart. Appl. Math. 40 (1983), 468-475.

[9] Friedman, A., Monotonic decay of solutions of parabolic equations with nonlocal boundary conditions, Quart. Appl. Math. 44 (1986), 401-407.

[10] Hess, P., Spatial homogeneity of stable solutions of some periodic-parabolic problems with Neumann boundary conditions, J. Differential Equations 
68 (1987), 320-331.

[11] Kawohl, B., Remarks on a paper by W.A. Day on a maximum principle under nonlocal boundary conditions, Quart. Appl. Math. 44 (1987), 751-752.

[12] Nakhusheva, Z.A., On a nonlocal problem for partial differential equations (in Russian), Diff. Uravn. 22 (1986), 171-174.

[13] Redheffer, R. and W. Walter, Das Maximumprinzip in unbeschränkten Gebieten für parabolische Ungleichungen mit Funktionalen, Math. Ann. 226 (1977), 155-170.

[14] Szarski, J., Strong maximum principle for nonlinear parabolic differentialfunctional inequalities in arbitrary domains, Ann. Polon. Math. 29 (1974), 207-217.

[15] Walter, W., Differential and integral inequalities, Springer Verlag, Berlin, Heidelberg, New York, 1970.

[16] Walter, W., On the strong maximum principle for parabolic differential equations, Proc. Edinburgh Math. Soc. 29 (1986), 93-96. 


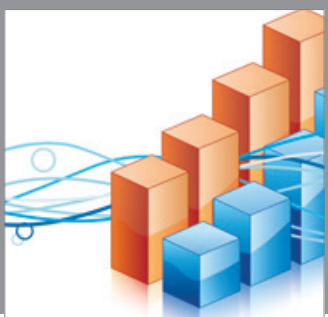

Advances in

Operations Research

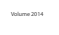

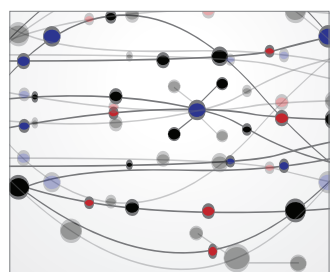

\section{The Scientific} World Journal
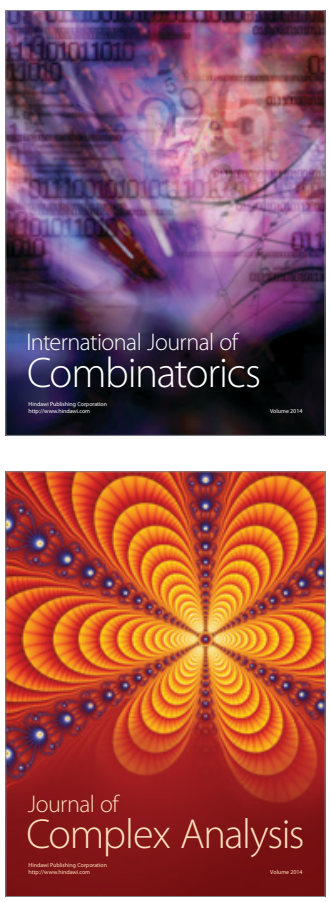

International Journal of

Mathematics and

Mathematical

Sciences
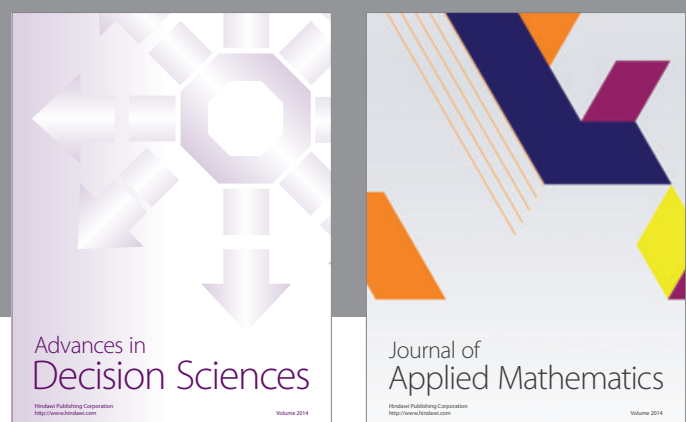

Journal of

Applied Mathematics
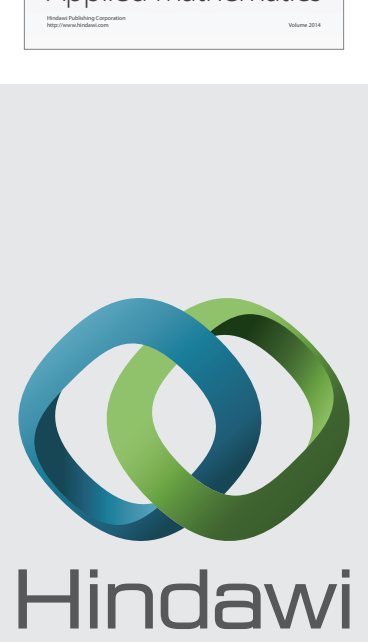

Submit your manuscripts at http://www.hindawi.com
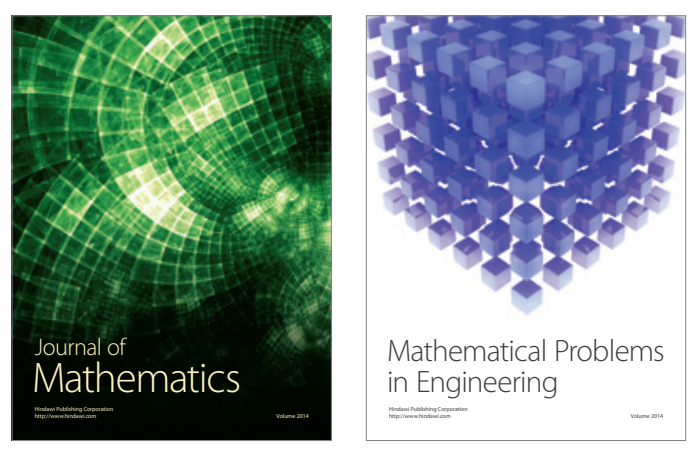

Mathematical Problems in Engineering
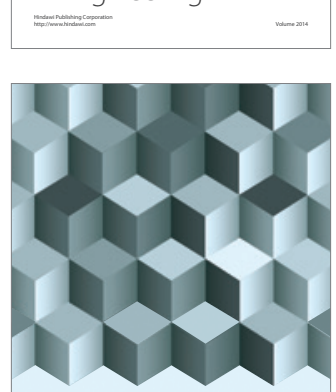

Journal of

Function Spaces
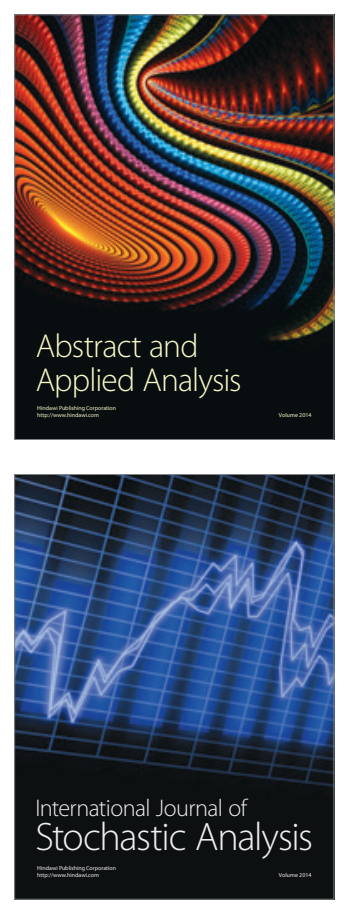

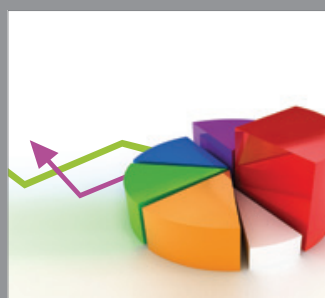

ournal of

Probability and Statistics

Promensencen
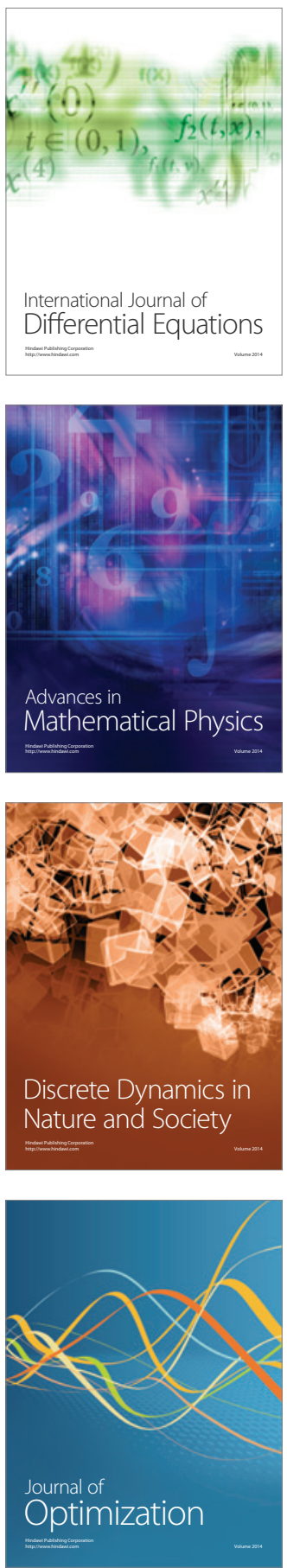